\title{
Adaptation et validation en langue française de mesures du climat scolaire
}

\author{
Jacques Richard \\ Université de Moncton \\ Jenny Coulombe \\ District scolaire 01, Nouveau-Brunswick
}

\section{Résumé}

L'objectif de cette étude est de vérifier la validité de la traduction et de l'adaptation française de deux questionnaires originellement de langue anglaise et visant à mesurer le climat scolaire, soit l'Elementary and Middle School Inventory of Classroom Environments (ICE) et le Climate of the Playground and Lunchroom Climate Questionnaire (PLCQ). Les versions françaises ont été administrées à un échantillon de 207 élèves francophones de la $6^{\mathrm{e}}$ à la $8^{\mathrm{e}}$ année. Les résultats révèlent des propriétés psychométriques satisfaisantes en ce qui concerne la validité de construit (structures factorielles) et la fidélité en termes de cohérence interne (coefficients alpha de Cronbach, corrélations items-total). Ces résultats s'avèrent relativement semblables à ceux rapportés par les auteurs des versions anglaises des instruments. Bien que des études plus approfondies pour confirmer la validité des instruments en langue française soient encore à faire, les données préliminaires permettent de croire que, dans leur forme actuelle, ceux-ci pourraient être utiles aux chercheurs et éducateurs qui désirent améliorer le climat scolaire de leur école en le rendant plus conforme aux attentes des élèves.

Mots clés : climat scolaire; traduction; validation; adaptation; Climate of the Playground and Lunchroom Climate Questionnaire (PLCQ); Elementary and Middle School Inventory of Classroom Environments (ICE)

\section{Abstract}

The purpose of this study is to verify the French translation and adaptation validity of two English questionnaires measuring school climate : The Elementary and Middle School Inventory of Classroom Environments (ICE) and the Climate of the Playground and Lunchroom Climate Questionnaire (PLCQ). The French versions of the questionnaires were administered to a sample of 207 francophone students from $6^{\text {th }}$ to $8^{\text {th }}$ grade. Results regarding the construct validity (factor analysis) and internal consistency reliability (Cronbach's alphas, item-total correlations) appear within the acceptable range. These results are similar to those reported for the original English-language versions. Although more studies are needed to further demonstrate the validity of these two French questionnaires, the preliminary results suggest that, in their present form, they could be useful to researchers and educators who want to bring the school climate in their community closer to the needs of students. 


\section{Introduction}

L'école est une communauté socialisante (Fraser, 1994; Leff, Power, Costigan et Manz, 2003; Peterson et Skiba, 2000). Elle occupe une place particulièrement importante dans le cheminement des jeunes issus des groupes désavantagés (Rutter, Maughan, Mortimore, Ouston et Smith, 1979), notamment les jeunes francophones en situation minoritaire. Les représentations linguistiques et culturelles véhiculées par le biais du milieu scolaire influent sur différentes facettes du vécu langagier des jeunes. Selon plusieurs, la nature du contexte socio-pédagogique peut contribuer (Cormier, 2005; Cormier, Pruneau, Rivard et Blain, 2004; Cormier, Pruneau, et Rivard, 2010) ou nuire (LeBlanc et Beaton, 2011) au rendement scolaire des francophones minoritaires. L'Association canadienne d'éducation de langue française (2006) accorde une importance cruciale au rôle de l'école dans le processus de construction identitaire du jeune écolier. En effet, le contexte éducatif offre au jeune francophone en milieu minoritaire des expériences, des modèles et des activités pédagogiques qui favorisent le regard critique à l'endroit des réalités ethnolinguistiques et l'engagement ethnolangagier (Allard, Landry et Deveau, 2005).

Bien sûr, pour avoir une influence positive sur le vécu des jeunes, le climat scolaire doit lui-même être perçu comme étant positif. Par conséquent, il est important de fournir une mesure qui évalue les dimensions d'un climat scolaire, adaptée aux jeunes francophones en milieu minoritaire. Or, à l'heure actuelle, il existe un nombre limité de recherches et d'instruments de mesure francophones du climat scolaire. Le but de cette étude est de pallier à cette lacune. Cette étude vise à effectuer la traduction et l'adaptation en langue française, ainsi que la validation d'instruments de mesure du climat scolaire qui rendent compte des dimensions essentielles du contexte sociopédagogique dans lequel baignent les jeunes qui fréquentent l'école intermédiaire.

\section{Le climat scolaire : définitions et dimensions}

Halpin et Croft (1963) ont conceptualisé le climat organisationnel de l'école comme étant la qualité d'un environnement qui découle d'une combinaison de facteurs physiques et sociaux. Le climat scolaire peut faire référence au climat de la classe ou au climat de l'école. Le climat au niveau de la classe se caractérise par les interactions partagées entre les élèves, ainsi qu'entre les élèves et leurs enseignants (Fisher, Grady et Fraser, 1995; Fraser, 1994). Le climat au niveau de l'école implique plus particulièrement la relation entre les enseignants, les autres 
membres du personnel et la direction de l'école. II fait aussi référence au climat social et disciplinaire présent partout à l'école, y compris dans la cour de récréation et à la cafétéria. Un lien relativement étroit existe entre les deux types de climat scolaire de façon à ce que le climat de l'école exerce une influence sur le climat de la classe (Fisher, Grady et Fraser, 1995).

Selon Moos (2002), les dimensions qui contribuent à un climat scolaire sain sont : (a) l'implication et l'intérêt des élèves, leur participation aux discussions de classe, (b) leur affiliation, comprenant le support réciproque et le plaisir de travailler ensemble, (c) le support des enseignants et leur intérêt au bien-être des élèves, (d) l'orientation de l'élève vers les tâches académiques et (e) l'organisation et l'ordre général. Des recherches plus récentes de Zullig, Huebner et Patton (2011) ont démontré que des variables de climat scolaire, telles que les relations élèvesenseignants positives, le support et la satisfaction académique, une discipline appropriée et le sentiment d'affiliation à l'école ont un impact significatif sur la satisfaction scolaire des élèves.

Les variations dans la réussite scolaire peuvent être attribuées plus globalement à l'organisation sociale, à la motivation face au milieu scolaire, au développement, à la stabilité et à l'autonomie personnelle, aux attitudes et aux comportements des enseignants, aux compétences académiques globales des élèves et à l'ensemble du climat social. De la même façon, les comportements inappropriés, l'abandon scolaire, la délinquance, la consommation de drogues peuvent aussi être liés, entre autres, au climat qui règne dans une école. Dans une étude auprès de 478 écoles françaises (Richard, Schneider et Mallet, 2012), on a compté moins d'épisodes d'intimidation scolaire (bullying) au sein des écoles ayant un climat scolaire plus favorable, particulièrement au niveau de la relation entre élèves et enseignants. Enfin, les niveaux d'inspiration et d'ambition face au climat d'apprentissage peuvent relever de la façon dont les élèves considèrent le climat scolaire comme contribuant à un environnement de soutien adéquat (Espelage et Swearer, 2004, 2010).

\section{Variables reliées au climat scolaire}

Haertel, Walberg et Haertel (1981) ont recensé douze publications scientifiques portant sur l'association entre le climat de la classe et divers résultats scolaires de 17805 élèves de quatre états américains. Les résultats obtenus mettent en évidence la forte relation entre la perception positive du climat de la classe par les élèves et des conséquences affectives et cognitives plus positives chez ces derniers. Des résultats similaires de McRobbie et Fraser (1993) appuient cette méta-analyse. Ces auteurs rapportent des relations positives entre la progression des apprentissages et la perception des élèves face à différentes dimensions du climat de la classe, telles que la cohésion, l'ouverture, l'intégration, la clarté des règles et l'organisation matérielle.

Les récréations scolaires peuvent aussi avoir un effet sur l'éducation et le développement de l'enfant (Leff, Power, Costigan et Manz, 2003). Elles permettent aux élèves de développer certaines compétences sociales, dont la capacité de s'adapter habilement aux demandes de leur école et à celles de leurs pairs. Les périodes de récréation permettent aussi aux enfants de participer à des activités physiques, ce qui contribue au développement de leurs habiletés motrices. Ces activités sont perçues comme un élément d'apprentissage dans la résolution de difficultés avec les pairs et dans le respect de certaines normes sociales. Les périodes de récréation ont un impact direct et bénéfique sur les capacités de l'enfant à avoir une meilleure attention et un meilleur apprentissage lors du retour en 
classe. De ce fait, les performances scolaires optimales sont obtenues lorsque l'enfant est capable d'une concentration attentive et ainsi, d'être relativement résistant aux distractions (Pellegrini et Bjorklund, 1996). Toutefois, la majorité des actions agressives, les blessures et la présence d'intimidation surviennent dans les cadres scolaires moins structurés, soit les périodes de récréation et les périodes de repas à la cafétéria (Leff, Power, Costigan et Manz, 2003; Pellegrini, 1995).

Les recherches effectuées par Pellegrini et Davis (1993) suggèrent la présence d'une forte corrélation entre l'activité physique modérée dans la cour de récréation et l'attention chez les élèves. Pour expliquer ce phénomène, ces auteurs font appel à la théorie selon laquelle tout individu a besoin de libérer le surplus d'énergie qui s'accumule durant une période prolongée d'activités sédentaires qui demandent de rester assis pendant une longue période de temps. Les éducateurs font souvent appel à cette théorie pour justifier la pratique de ménager des périodes de récréation afin de libérer les élèves de cette énergie excédante (Pellegrini, 1995). Même si des recherches plus approfondies doivent être faites pour démontrer la validité de cette théorie, certaines observations de l'étude de Pellegrini et Davis (1993) suggèrent la nécessité d'un changement de niveau d'activité chez les élèves. La présence de périodes de récréation demeure donc importante dans la mesure où ces périodes de jeux restent à un niveau moyen d'excitation.

Les comportements de l'enfant durant les périodes de récréation et lors des repas à la cafétéria semblent être affectés par la façon dont les membres du personnel de l'école gèrent ces activités. La recherche effectuée par Leff, Power, Costigan et Manz (2003) indique que l'organisation d'activités récréatives structurées par le personnel scolaire durant la période de récréation est associée à une plus grande participation des élèves dans ces activités et donc augmente la probabilité pour les élèves d'en retirer les bienfaits qui y sont associés. De plus, la formation de superviseurs dans le but de renforcer activement les comportements prosociaux, entraîne une diminution marquée de la présence de comportements d'agression entre élèves tels que se donner des coups de pied et des coups de poing.

L'importance du climat scolaire dans la réussite scolaire et le comportement des élèves est documentée depuis les études pionnières de Michael Rutter et ses collègues (Rutter, Maughan, Mortimore, Ouston, Smith, 1979; Mortimore, 1995) auprès d'élèves de la Grande-Bretagne. Ces études, tout comme celles plus récentes mentionnées précédemment, démontrent l'importance de ne pas négliger la contribution du climat scolaire au bien-être des élèves, à leur développement social et émotionnel, ainsi qu'au développement des habiletés cognitives et académiques nécessaires à leur réussite scolaire. Toutefois, la grande majorité de ces études ont été effectuées auprès d'élèves anglophones issus d'un milieu majoritaire. Ainsi, dans l'optique de contribuer aux travaux de recherche futurs auprès des élèves francophones, y inclus les jeunes francophones en situation minoritaire, il convient de doter les chercheurs d'outils permettant la mesure du climat scolaire auprès de ces derniers.

\section{Objectifs spécifiques de l'étude}

Bien qu'il existe quelques instruments de mesure francophones du climat scolaire, tels que le Questionnaire sur l'environnement socio-éducatif (QES) développé par Janosz, Georges et Parent (1998), l'Échelle pour la mesure de l'environnement du cours (EMEC) par Diaz (1983) et le Questionnaire d'évaluation du climat de la classe (ÉEC) traduit et adapté par Tremblay et Desmarais-Gervais (cité dans Cossette, Potvin, Marcotte, Fortin, Royer et Leclerc, 
2004), ces instruments ne sont pas adaptés aux élèves francophones des niveaux scolaires intermédiaires, c'est-àdire de la fin du primaire et du début du secondaire, et ils n'évaluent pas le climat qui règne dans la cour de récréation et à la cafétéria. Ainsi, cette étude vise à combler cette lacune. L'objectif est de vérifier la validité de la traduction et de l'adaptation en langue française de deux questionnaires développés en langue anglaise : l'Elementary and Middle School Inventory of Classroom Environments (ICE - Students' Perceived Classroom - Students' Preferred Classroom) (Sinclair et Fraser, 2002) et le Climate of the Playground and Lunchroom Climate Questionnaire (PLCQ) (Leff, Power, Costigan et Manz, 2003).

L'ICE est un instrument de mesure du climat de la salle de classe qui possède des propriétés psychométriques satisfaisantes (Sinclair et Fraser, 2002) et qui innove dans la mesure où il se présente sous deux formes parallèles permettant aux chercheurs d'évaluer chez les élèves la perception actuelle et la perception souhaitée du climat de la classe. Sinclair et Fraser (2002) ont validé l'ICE à partir d'une population de 745 élèves de niveau intermédiaire. Pour chacune des deux formes du questionnaire, une analyse factorielle et une analyse d'items ont permis d'établir la validité de 20 items distribués dans quatre échelles: Collaboration (4 items), Empathie de l'enseignant (8 items), Participation (4 items) et Orientation vers les tâches (4 items). L'estimation de la fidélité a ensuite été faite à deux niveaux : celui de la moyenne obtenue par l'ensemble de la classe et celui des scores individuels des élèves et ce, pour les quatre échelles de l'ICE et les deux formes du questionnaire. La cohérence interne au niveau de la moyenne de classe a été mesurée par les coefficients alpha de Cronbach, lesquels se situent entre 0,64 et 0,94 pour la perception actuelle et entre 0,60 et 0,90 pour la perception souhaitée. Au niveau des élèves individuellement, les coefficients alpha se situent entre 0,54 et 0,86 pour la perception actuelle et entre 0,61 et 0,82 pour la perception souhaitée. La fidélité estimée au niveau des élèves individuellement est légèrement plus faible que celle au niveau de l'ensemble de la classe, mais tout de même satisfaisante pour des échelles de mesure relativement courtes.

Pour ce qui est du PLCQ, selon Leff, Power, Costigan et Manz (2003), une force majeure réside dans sa sensibilité à la dynamique présente dans le cadre des périodes de récréation et lors des périodes de repas à la cafétéria. Le $P L C Q$, développé grâce à la participation de plusieurs membres du personnel de diverses écoles, fournit des informations sur le comportement des surveillants ainsi que des élèves dans des contextes scolaires peu structurés (Leff, Power et Goldstein, 2004). De plus, le PLCQ, qui s'administre en une dizaine de minutes, n'interfère pas avec les heures de cours des enseignants et des élèves. D'un point de vue pratique, le $P L C Q$ a été développé afin de fournir des données pouvant être utilisées dans la planification de programmes de prévention ou d'intervention face à l'intimidation scolaire.

Le $P L C Q$ contient 23 items au total. Une analyse factorielle du $P L C Q$ à partir des résultats obtenus à la suite à l'administration du questionnaire auprès de 122 membres du personnel de 12 écoles élémentaires, 2 écoles intermédiaires et 3 écoles mixtes, élémentaires et intermédiaires, révèle deux dimensions majeures: 1) Structuration de la surveillance et des activités (14 items), et 2) Collaboration entre les membres du personnel de l'école (9 items) (Leff, Power, Costigan et Manz, 2003). Les deux facteurs sont modérément corrélés entre eux ( $r=0,56)$. La cohérence interne, calculée à l'aide du coefficient alpha, est de 0,84 pour chacune des deux dimensions ou échelles. 


\section{Méthodologie}

\section{Participants et procédure d'administration des instruments de mesure}

Le processus de validation des versions françaises de l'ICE et du PLCQ se veut parallèle à celui adopté par les auteurs des versions originales anglaises afin de faciliter les comparaisons. Signalons que pour I'ICE en particulier, même si l'instrument s'adresse à la fois aux élèves des niveaux élémentaire et intermédiaire, la validation n'a été faite qu'au niveau intermédiaire. Ainsi, les participants à notre étude sont des élèves de trois écoles offrant des programmes de la $6^{e}$ à la $8^{e}$ année de deux districts scolaires francophones du Nouveau-Brunswick. Dix classes ont été choisies au hasard dans ces écoles et selon les informations obtenues de leur district scolaire respectif, celles-ci sont situées dans des milieux ruraux et urbains hétérogènes en termes de statut socio-économique. L'étude a été approuvée par le Comité d'éthique de la recherche de l'Université de Moncton et le consentement éclairé des élèves et de leurs parents a été sollicité par écrit. Sur les 250 élèves possibles, un total de 207 élèves a accepté de participer à la recherche, dont 64 élèves de la 6 e année, 58 élèves de la 7 e année et 85 élèves de la 8 e année.

En ce qui a trait à l'administration des instruments de mesure, les participants ont été regroupés dans différentes salles de classe. Chaque groupe a reçu un rappel des principes de confidentialité et du droit de se retirer de l'étude à tout moment. Après avoir indiqué les objectifs de l'étude et expliqué les consignes, l'administration des questionnaires a été surveillée par des étudiants gradués en psychologie. S'il était nécessaire, par exemple dans le cas d'élèves avec difficultés en lecture ou en compréhension, les surveillants pouvaient offrir de l'aide individuelle en lisant les questions et les choix de réponses à haute voix, ou en donnant la définition d'un mot.

\section{Traduction et adaptation des instruments de mesures}

En se basant sur les recommandations de la Commission internationale des tests (voir Hambleton, 1994), trois étapes ont été identifiées pour l'adaptation des mesures. La première étape a consisté à faire traduire chaque directive et item des questionnaires par six traducteurs. La Commission internationale des tests suggère que les traducteurs soient familiers et aient de l'expérience avec les deux cultures (la langue source et la langue ciblée), le sujet évalué par les questionnaires (le climat scolaire) et la procédure nécessaire pour traduire un instrument de mesure (Hambleton, 1994). La deuxième étape a impliqué la constitution d'un comité composé de deux professeurs et de trois étudiantes en psychologie, tous familiers avec la psychométrie, la procédure à suivre pour adapter un test, les caractéristiques et le langage des enfants d'âge scolaire, ainsi que les langues et les cultures anglaise et française. Les membres du comité, natifs de diverses régions du Québec et du Nouveau-Brunswick et ayant tous le français comme langue maternelle, devaient vérifier l'exactitude de la traduction de chaque item des questionnaires en termes de sémantique, connotation, cohérence, lisibilité, intérêt intrinsèque, familiarité et difficulté du contenu de l'item (Hambleton, 1994; Laveault et Grégoire, 2002). Le comité devait choisir la meilleure traduction proposée par les 6 traducteurs et pouvait apporter les changements jugés nécessaires lorsque la traduction en langue française ne leur semblait pas satisfaisante (Vallerand, 1989). Après avoir compilé l'ensemble des items traduits, le nouveau questionnaire a été soumis en troisième étape à une évaluation de surface par une enseignante de 6e année, un professeur de français, ainsi qu'une psychologue pour enfants, que nous estimions capables de juger de la qualité du français et de la pertinence du questionnaire pour la population cible. 


\section{Instruments de mesure}

L'Inventaire de l'environnement de classe d'écoles élémentaires et intermédiaires, classe perçue et classe préférée (IEC - Perception de ta classe - La classe que tu aimerais avoir) vise à évaluer la perception actuelle et la perception souhaitée du climat de la classe par des élèves de niveaux élémentaire et intermédiaire. L'instrument contient les mêmes échelles et items que la version originale, c'est-à-dire 20 items distribués dans quatre échelles : Collaboration (4 items, p. ex., « Je travaille bien avec les autres élèves de la classe »), Empathie de l'enseignant (8 items, p. ex., «Mon enseignant(e) veut que je réussisse bien en classe »), Participation (4 items, p. ex., " Je réponds aux questions en classe ») et Orientation vers les tâches (4 items, p. ex., « Je suis attentif en classe »). Les réponses aux items s'expriment sur une échelle de type Likert en trois points : $1=$ rarement, $2=$ parfois et $3=$ souvent. Cet instrument se divise en deux parties ou formes parallèles dans lesquelles se retrouvent des items portant sur des contenus similaires. La première partie mesure la perception actuelle des élèves du climat de leur classe et contient des items tels que « Mon enseignant(e) m'aime bien ». Dans la deuxième partie, les items sont formulés de façon à faire ressortir la perception des élèves de ce que serait pour eux un climat de classe idéal comme, par exemple « Je voudrais que mon enseignant(e) m'aime bien ».

Pour sa part, l'Inventaire du climat de la cour de récréation et de la cafétéria (ICRC) évalue le climat scolaire qui règne pendant les périodes de récréation et lors des repas à la cafétéria. Comme l'instrument original a été conçu pour être administré aux adultes effectuant la supervision durant les périodes scolaires moins structurées, dans le cadre de notre étude, une adaptation a été effectuée afin de le rendre accessible aux élèves des niveaux scolaires intermédiaires. Dans cette optique, nous avons éliminé les 9 items mesurant la dimension «Collaboration entre les membres du personnel de l'école », les élèves n'ayant pas les connaissances ou les expériences nécessaires pour y répondre d'une manière appropriée (p. ex., "Staff members work well together and as a team »). Les 14 items que nous avons conservés font partie de la dimension «Structuration de la surveillance et des activités » obtenue dans l'analyse initiale de Leff, Power, Costigan et Manz (2003). Un exemple d'item se lit comme suit : « Les surveillant(e)s font respecter les règlements dans la cour de récréation ».

\section{Procédures d'analyse des données}

Aux fins de validation des instruments de mesure traduits et adaptés en français, nous avons repris les méthodes d'analyse statistique utilisées par les auteurs des versions originales anglaises, soit la procédure d'analyse factorielle avec rotation varimax, de même que le calcul du coefficient alpha de Cronbach et des corrélations itemstotal. Cette façon de procéder permet de comparer nos résultats avec les leurs. L'analyse factorielle est une procédure utilisée pour déterminer la validité hypothético-déductive ou de construit d'un test, tandis que le coefficient alpha de Cronbach et les corrélations entre les items et le score total sont des mesures de fiabilité donnant une indication de la cohérence interne d'un test (Anastasi et Urbina, 1997).

\section{Résultats}

La présentation des résultats se concentre d'abord sur la validité de construit des instruments de mesure du climat scolaire traduits et adaptés, soit l'IEC - Perception de ta classe - La classe que tu aimerais avoir, et l'ICRC. II 
s'agit d'en examiner la structure factorielle et d'établir un parallèle avec les instruments originaux de Sinclair et Fraser (2002) et de Leff, Power, Costigan et Manz (2003). Nous vérifions ensuite la fiabilité des instruments.

\section{Structure factorielle : IEC - Perception de ta classe}

L'analyse factorielle en composantes principales avec rotation varimax de la partie Perception de ta classe de l'IEC révèle une structure à quatre facteurs expliquant $54 \%$ de la variance. Le tableau 1 rapporte les résultats de cette analyse. Le premier facteur « Collaboration » regroupe les 4 items 1, 6, 11 et 16, avec des saturations qui varient de 0,56 à 0,67 . Pour le deuxième facteur, "Orientation vers les tâches " les items se répartissent en deux groupements. On retrouve les 4 items 2, 7, 12 et 17 dont les saturations se situent de 0,53 à 0,84. Cependant, 5 autres items $(3,8,18,14$ et 15) ont aussi des saturations allant de 0,30 à 0,39 sur ce facteur. Ces derniers items ont cependant des saturations plus élevées dans les troisième et quatrième facteurs. En effet, le troisième facteur «Participation » regroupe les items 3,8, 13 et 18 dont les saturations varient de 0,49 à 0,81. L'item 5 y a une saturation de 0,38. Enfin le quatrième facteur « Empathie de l'enseignant » inclut 8 items $(4,5,9,10,14,15,19,20)$ dont les saturations varient de 0,58 à 0,76. Si l'on considère seulement les saturations les plus élevées (de 0,49 et plus) la totalité des items se regroupent exactement sous les mêmes dimensions que ceux de la version originale de Sinclair et Fraser (2002).

Tableau 1

Résultats de l'analyse factorielle en composante principale avec rotation varimax des 20 items de l'IEC Perception de ta classe

\begin{tabular}{ccccc}
\hline Items & Collaboration & $\begin{array}{c}\text { Orientation vers } \\
\text { les tâches }\end{array}$ & Participation & $\begin{array}{c}\text { Empathie } \\
\text { de l'enseignant }\end{array}$ \\
\hline 1 & 0,67 & - & - & - \\
6 & 0,65 & - & - & - \\
11 & 0,63 & - & - & - \\
16 & 0,56 & - & - & - \\
2 & - & 0,76 & - & - \\
7 & - & 0,59 & - & - \\
12 & - & 0,53 & - & - \\
17 & - & 0,84 & 0,63 & - \\
3 & - & 0,35 & 0,64 & - \\
8 & - & 0,39 & 0,81 & - \\
13 & - & - & 0,49 & 0,65 \\
18 & - & 0,38 & - & 0,58 \\
4 & - & - & 0,38 & 0,67 \\
5 & - & - & - & 0,73 \\
9 & - & - & - & 0,69 \\
10 & - & - & - & 0,72 \\
14 & - & 0,31 & - & 0,72 \\
15 & - & 0,30 & - & 0,76 \\
19 & - & - & - & - \\
20 & - & - & & - \\
\hline
\end{tabular}

Seulement les saturations $\geq 0,30$ sont présentées. 


\section{Structure factorielle : IEC - La classe que tu aimerais avoir}

Pour ce qui est des 20 items de la deuxième partie de l'IEC portant sur La classe que tu aimerais avoir, l'analyse en composantes principales donne, encore une fois, une structure à quatre facteurs expliquant $61 \%$ de la variance (voir tableau 2). Une majorité d'items se répartissent de la même manière que ceux de la première partie de I'IEC. Pour le premier facteur « Collaboration » on retrouve les trois items 6, 11 et 16, avec des saturations allant de 0,30 à 0,85 . L'item 1 se classe maintenant dans le deuxième facteur "Orientation vers les tâches » avec une saturation de 0,85 . Pour ce deuxième facteur, on retrouve encore les trois items 2,12 et 17 avec des saturations de 0,43 à 0,80 . L'item 7 ne fait plus partie de ce facteur. Cinq autres items $(3,4,5,9$ et 10) ont des saturations allant de 0,30 à 0,53 sur ce facteur. Ces derniers items, sauf l'item 4, ont des saturations plus élevées dans les $3^{\mathrm{e}}$ et 4e facteurs. Le troisième facteur «Participation » regroupe encore les items 3, 8, 13, 18 et 5 dont les saturations varient de 0,55 à 0,74. II s'y ajoute les items 4, 7,19 et 20 avec des saturations allant de 0,30 à 0,57. Enfin le quatrième facteur « Empathie de l'enseignant» inclut 7 des 8 items de la première partie de l'ICE $(4,9,10,14,15,19,20)$ dont les saturations varient de 0,44 à 0,79 . Quatre autres items $(7,12,16$ et 17) y ont aussi des saturations variant de 0,34 à $0,47)$. Ces résultats font donc ressortir une structure factorielle moins parfaite, mais qui comporte plusieurs similarités à celle obtenue avec l'IEC - Perception de ta classe. Cette structure laisse toutefois entendre que les variables médiatrices en jeu dans la perception du climat actuel de la classe interviennent de façon quelque peu différente dans la perception du climat souhaité. Pour ce qui est de la comparaison avec l'étude de Sinclair et Fraser (2002), ces

Tableau 2

\section{Résultats de l'analyse factorielle en composante principale avec rotation varimax des 20 items de l'IEC - La} classe que tu aimerais avoir

\begin{tabular}{rcccc}
\hline Items & Collaboration & $\begin{array}{c}\text { Orientation vers } \\
\text { les tâches }\end{array}$ & Participation & $\begin{array}{c}\text { Empathie } \\
\text { de l'enseignant }\end{array}$ \\
\hline 1 & - & 0,85 & - & - \\
6 & 0,82 & - & - & - \\
11 & 0,85 & - & - & - \\
16 & 0,30 & 0,53 & - & 0,42 \\
2 & - & 0,80 & - & - \\
7 & - & - & 0,57 & 0,34 \\
12 & - & 0,43 & - & 0,47 \\
17 & - & 0,69 & - & 0,41 \\
3 & - & 0,53 & 0,56 & - \\
8 & - & - & 0,74 & - \\
13 & - & - & 0,72 & - \\
18 & - & - & 0,71 & - \\
4 & - & 0,51 & 0,37 & 0,44 \\
5 & - & 0,30 & 0,55 & - \\
9 & - & 0,45 & - & 0,53 \\
10 & - & 0,33 & - & 0,55 \\
14 & - & - & - & 0,79 \\
15 & - & - & - & 0,67 \\
19 & - & - & 0,34 & 0,73 \\
20 & - & - & 0,30 & 0,74 \\
\hline
\end{tabular}

Seulement les saturations $\geq 0,30$ sont présentées. 
auteurs ne présentent pas le détail de la structure factorielle de cette partie de l'instrument et se limitent à affirmer qu'ils ont obtenus une structure factorielle similaire à celle de l'IEC - Perception de ta classe.

\section{Structure factorielle : ICRC- Version pour l'élève}

L'analyse en composantes principales avec rotation varimax des 14 items de l'ICRC constituant la version pour les élèves donne une structure à deux dimensions expliquant $43 \%$ de la variance (voir tableau 3). Nous avons nommé ces deux dimensions «Structure » et « Activités ». La dimension « Structure » contient les items 2, 3, 5, 8, 10, 13 et 14 , avec des saturations qui varient de 0,64 à 0,76. L'item 4 y a une saturation de 0,40. Ces items portent sur des contenus reliés principalement à l'application des règlements disciplinaires (p. ex., «Les surveillant(e)s font respecter les règlements dans la cour de récréation »). La deuxième dimension «Activités » regroupe les items 1, 4,6, $7,9,11$ et 12 , avec des saturations qui varient de 0,43 à 0,69. Ces items portent davantage sur les activités offertes aux élèves pendant les périodes de récréations (p. ex., « II y a plusieurs jeux auxquels les élèves peuvent jouer dans la cour de récréation»). L'item 4 («Les surveillant(e)s de la cafétéria et de la cour de récréation ont une attitude positive envers les élèves ») a la particularité de saturer sous chacune des deux dimensions. Dans la version originale de Leff, Power, Costigan et Manz (2003), tous les 14 items se retrouvaient au sein de la dimension « Structuration de la surveillance et des activités ». Cependant, la nature des deux dimensions retrouvées à la suite des modifications apportées au questionnaire reflètent quand même les construits théoriques décrits par ces auteurs.

Tableau 3

Résultats de l'analyse factorielle en composante principale avec rotation varimax des 14 items de l'ICRF

\begin{tabular}{rcc}
\hline Items & Structure & Activités \\
\hline 1 & - & 0,69 \\
4 & 0,40 & 0,44 \\
6 & - & 0,49 \\
7 & - & 0,43 \\
9 & - & 0,69 \\
11 & - & 0,65 \\
12 & - & 0,56 \\
2 & 0,66 & - \\
3 & 0,66 & - \\
5 & 0,64 & - \\
8 & 0,70 & - \\
10 & 0,71 & - \\
13 & 0,66 & - \\
14 & 0,76 & - \\
\hline
\end{tabular}

Seulement les saturations $\geq 0,30$ sont présentées.

\section{Cohérence interne des instruments}

Pour l'échelle IEC - Perception de ta classe, les coefficients alpha de Cronbach pour les dimensions Collaboration, Orientation vers les tâches, Participation et Empathie de l'enseignant sont respectivement de 0,49, 0,74, 0,74 et 0,85 (voir tableau 4). Ceux-ci sont très similaires à ceux obtenus avec l'instrument anglais original, Sinclair et Fraser (2002) ayant rapporté des coefficients de 0,54,0,70,0,60 et 0,86 pour les mêmes dimensions. En ce qui a trait aux corrélations items-total, bien que deux items (11 et 16) aient des corrélations plus faibles que 0,30, aucun des 20 
items n'a une corrélation item-total négative. Les corrélations items-total varient de 0,19 à 0,66. Ces résultats sont relativement satisfaisants et indiquent une cohérence interne acceptable pour chaque dimension.

Tableau 4

Corrélations items-total et coefficients alpha de Cronbach pour les quatre dimensions de I'IEC -

Perception de ta classe $(\mathrm{N}=\mathbf{2 0 7})$

\begin{tabular}{|c|c|c|c|}
\hline Dimensions & Items & $\begin{array}{c}\text { Corrélations } \\
\text { Item-total }\end{array}$ & Alpha de Cronbach \\
\hline \multirow[t]{5}{*}{ Collaboration } & 1 & 0,39 & \\
\hline & 6 & 0,36 & \\
\hline & 11 & 0,19 & \\
\hline & 16 & 0,25 & \\
\hline & & & 0,49 \\
\hline \multicolumn{4}{|l|}{ Orientation } \\
\hline \multirow[t]{5}{*}{ vers les tâches } & 2 & 0,56 & \\
\hline & 7 & 0,50 & \\
\hline & 12 & 0,42 & \\
\hline & 17 & 0,66 & \\
\hline & & & 0,74 \\
\hline \multirow[t]{5}{*}{ Participation } & 3 & 0,61 & \\
\hline & 8 & 0,54 & \\
\hline & 13 & 0,52 & \\
\hline & 18 & 0,46 & \\
\hline & & & 0,74 \\
\hline \multicolumn{4}{|l|}{ Empathie de } \\
\hline \multirow[t]{9}{*}{ L'enseignant } & 4 & 0,57 & \\
\hline & 5 & 0,52 & \\
\hline & 9 & 0,56 & \\
\hline & 10 & 0,58 & \\
\hline & 14 & 0,63 & \\
\hline & 15 & 0,65 & \\
\hline & 19 & 0,59 & \\
\hline & 20 & 0,65 & \\
\hline & & & 0,85 \\
\hline
\end{tabular}

Pour l'échelle IEC - La classe que tu aimerais avoir, les coefficients alpha de Cronbach pour les dimensions Collaboration, Orientation vers les tâches, Participation et Empathie de l'enseignant sont respectivement de 0,52, 0,78, 0,78 et 0,86 (voir tableau 5). Sinclair et Fraser (2002) avaient rapporté des coefficients de 0,61, 0,82, 0,71 et de 0,71 pour ces dimensions. On retrouve encore deux items (1 et 11) qui ont des corrélations items-total plus faibles que 0,30 et aucun des 20 items n'a de corrélation item-total négative. Les corrélations items-total varient de 0,14 à 0,69. Signalons que pour les deux versions de l'instrument, la dimension Collaboration est celle qui s'avère la moins fiable, les coefficients se situant en dessous du seuil d'acceptabilité de 0,70. En ce qui concerne L'ICRC - Version pour l'élève, les modifications apportées à la version originale anglaise du PLCQ validée par Leff, Power, Costigan et Manz (2003) empêche toute comparaison. Néanmoins, pour la version française, le coefficient alpha de Cronbach obtenu pour la dimension Structure est de 0,81 alors que celui pour la dimension Activités est de 0,69 (voir tableau 6). Ces résultats suggèrent une cohérence interne acceptable pour l'ICRC. Tous les items obtiennent une corrélation itemtotal positive et seul l'item 7 possède une corrélation inférieure à 0,30 . Les corrélations item-total varient de 0,24 à 0,62 . 
Tableau 5

Corrélations items-total et coefficients alpha de Cronbach pour les quatre dimensions de l'IEC -

La classe que tu aimerais avoir $(\mathrm{N}=207)$

\begin{tabular}{|c|c|c|c|}
\hline Dimensions & Items & $\begin{array}{l}\text { Corrélations } \\
\text { Item-total }\end{array}$ & Alpha de Cronbach \\
\hline \multirow[t]{5}{*}{ Collaboration } & 1 & 0,21 & \\
\hline & 6 & 0,57 & \\
\hline & 11 & 0,14 & \\
\hline & 16 & 0,42 & \\
\hline & & & 0,52 \\
\hline \multicolumn{4}{|l|}{ Orientation } \\
\hline \multirow[t]{5}{*}{ vers les tâches } & 2 & 0,60 & \\
\hline & 7 & 0,54 & \\
\hline & 12 & 0,55 & \\
\hline & 17 & 0,65 & \\
\hline & & & 0,78 \\
\hline \multirow[t]{5}{*}{ Participation } & 3 & 0,61 & \\
\hline & 8 & 0,64 & \\
\hline & 13 & 0,54 & \\
\hline & 18 & 0,56 & \\
\hline & & & 0,78 \\
\hline \multicolumn{4}{|l|}{ Empathie de } \\
\hline \multirow[t]{9}{*}{ l'enseignant } & 4 & 0,64 & \\
\hline & 5 & 0,48 & \\
\hline & 9 & 0,64 & \\
\hline & 10 & 0,67 & \\
\hline & 14 & 0,60 & \\
\hline & 15 & 0,65 & \\
\hline & 19 & 0,68 & \\
\hline & 20 & 0,69 & \\
\hline & & & 0,86 \\
\hline
\end{tabular}

Tableau 6

Corrélations items-total pour les deux dimensions de l'ICRC ( $N=207)$

\begin{tabular}{llll}
\hline Dimensions & Items & $\begin{array}{l}\text { Corrélations } \\
\text { Item-total }\end{array}$ & Alpha de Cronbach \\
\hline Activités & 1 & 0,45 & \\
& 4 & 0,38 & \\
& 6 & 0,30 & \\
& 7 & 0,24 & \\
9 & 0,57 & \\
& 11 & 0,41 & \\
Structure & 12 & 0,43 & \\
& 2 & 0,52 & \\
& 3 & 0,54 & \\
& 5 & 0,50 & \\
& 8 & 0,55 & www.reefmm.org
\end{tabular}




\section{Discussion}

Cette étude visait à vérifier la validité de la traduction et de l'adaptation en langue française de l'Elementary and Middle School Inventory of Classroom Environments (ICE - Students' Perceived Classroom et ICE - Students' Preferred Classroom), ainsi que du Climate of the Playground and Lunchroom Climate Questionnaire. En suivant une démarche de traduction et de validation transculturelle à plusieurs étapes, les versions françaises, notamment I'Inventaire de l'environnement de classe d'écoles élémentaires et intermédiaires, classe perçue et classe préférée (IEC - Perception de ta classe et IEC - La classe que tu aimerais avoir), et I'Inventaire du climat de la cour de récréation et de la cafétéria (ICRC-Version pour l'élève) possèdent des structures factorielles similaires aux questionnaires anglais originaux développés par Sinclair et Fraser (2002), ainsi que par Leff, Power, Costigan et Manz (2003), respectivement. Pour le questionnaire IEC, nous obtenons un instrument contenant quatre dimensions: Collaboration, Orientation vers les tâches, Participation et Empathie de l'enseignant, expliquant $54 \%$ de la variance. Pour l'ICRC, l'analyse fait ressortir une structure à deux dimensions, Structure et Activités, qui vont de pair avec le construit théorique présenté par Leff, Power, Costigan et Manz (2003). Les coefficients alpha de Cronbach et les corrélations items-total obtenus militent aussi en faveur de la fiabilité de ces instruments en termes de cohérence interne. Ces caractéristiques psychométriques préliminaires démontrent une validité et une fidélité satisfaisantes pour les deux instruments. Étant donné que les versions françaises étudiées ici demeurent fidèles à l'essence des questionnaires anglophones originaux, leur utilisation pourrait être envisagée autant pour l'intervention visant à améliorer le climat scolaire que pour l'étude de ce climat tel que perçu et souhaité par les élèves des niveaux intermédiaires dans les écoles francophones en milieu minoritaire.

Dans le domaine de l'intervention, une piste à explorer découle des études sur la correspondance entre la perception actuelle du climat scolaire et les attentes concernant le climat désiré. On sait en effet depuis longtemps que les élèves réussissent mieux et démontrent une meilleure attitude lorsqu'il y a un haut niveau de concordance entre le climat perçu et le climat désiré (Allen et Fraser, 2007; Fraser, 1989; 1994; Fraser et Fisher, 1983; Hunt, 1975). Les travaux plus récents de Fraser et ses collègues (Aldridge, Fraser et Sebela, 2004; Sinclair et Fraser, 2002) montrent qu'il est possible d'apporter un changement au climat de la classe afin que les perceptions actuelles et les attentes des élèves se rapprochent de plus en plus les unes des autres. Pour ce faire, ces auteurs recommandent de suivre un processus de recherche-action composé de plusieurs étapes impliquant 1) l'évaluation des perceptions actuelles et des attentes des élèves en ce qui a trait au climat de la classe ; 2) le partage des résultats avec les enseignants; 3) la mise en place d'un plan d'action pour améliorer le climat de la classe; 4) la cueillette de données qualitatives chez les élèves à propos des changements au niveau de la classe, des activités et de l'enseignant; 5) des rencontres hebdomadaires entre enseignants et chercheurs concernant les différentes techniques utilisées et 6 ) la réévaluation des perceptions du climat de la classe des élèves. Les enseignants qui reçoivent un soutien et une formation appropriés peuvent faire usage des réactions et du point de vue des élèves afin d'améliorer la qualité du climat de leur classe et, par ricochet, la réussite de leurs élèves (Sinclair et Fraser, 2002).

Il est permis de croire qu'une telle approche de modification du climat scolaire peut s'appliquer aux membres du personnel scolaire qui ont la responsabilité des activités moins structurées pendant les récréations et à la cafétéria. 
En effet, plusieurs recherches font ressortir l'intérêt d'examiner davantage l'effet de l'amélioration du climat scolaire sur le bien-être des élèves et la diminution des occasions d'intimidation (Ciucci et Smorti, 1998; Melton, Limber, Cunningham, Osgood, Chambers, Flerx, Henggeler et Nation, 1998; Stevens, De Bourdeaudhuij et Van Oost, 2000). Ces travaux misent sur l'étroite collaboration du personnel scolaire en vue d'implanter une politique claire contre les comportements d'intimidation peu importe que ceux-ci se présentent dans la salle de classe, dans la cour ou ailleurs dans l'école, sur le chemin de l'école ou même au sein de la communauté. La prise en compte du point de vue des élèves sur la qualité de la structuration de la surveillance et des activités qui leur sont offertes en dehors de la salle de classe peut certainement contribuer à l'élaboration de politiques mieux adaptées à leurs besoins, tout comme il apporte un complément d'informations important pour élargir la compréhension des facteurs en jeu dans le développement d'un climat d'école positif.

En ce qui a trait à la recherche sur le climat scolaire en milieu minoritaire francophone, il n'existe pas, à notre connaissance, de recherche empirique qui permettrait de connaitre comment les élèves des écoles francophones perçoivent le climat de leur école, ni quelles sont leurs attentes face à ce climat. Sans doute, mieux connaitre ces réalités pourrait s'avérer un atout et alimenter les recherches entourant le modèle conceptuel de Landry et ses collaborateurs (Landry, Allard, Deveau et Bourgeois, 2005) sur le rôle de l'école dans le processus de socialisation langagière. En effet, il est permis de faire l'hypothèse qu'un climat scolaire positif qui met l'accent, entre autres, sur la collaboration, l'orientation vers les tâches et la participation en salle de classe, dans la cour de récréation et aux heures des repas aurait un impact positif dans la socialisation langagière du jeune élève francophone évoluant en situation minoritaire.

Signalons enfin que la présente étude ne permet pas de conclure d'une manière définitive sur la validité des instruments traduits et adaptés. En effet, la cohérence interne de quelques échelles n'est pas aussi élevée que souhaitée et nous n'avons pas de données par rapport à la stabilité temporelle des outils. De plus, l'échantillon d'élèves ayant participé à cette étude se limite au niveau intermédiaire et n'est pas nécessairement représentatif de l'ensemble de la population francophone en milieu minoritaire canadien. Par conséquent, malgré des résultats préliminaires encourageants, l'utilisation de ces outils et l'interprétation des résultats qui en découlent doivent se faire avec prudence.

\section{Conclusion}

L'importance largement reconnue du contexte socio-pédagogique sur le bien-être, le rendement scolaire et la construction identitaire des élèves francophones en situation minoritaire (Cormier, 2005; Cormier, Pruneau, Rivard, et Blain, 2004; Cormier, Pruneau, et Rivard, 2010; LeBlanc et Beaton, 2011), de même que l'étroite relation établie entre la perception positive du climat scolaire par les élèves et les conséquences tout aussi positives sur leur développement cognitif, affectif, social et comportemental (Haertel, Walberg et Haertel, 1981; McRobbie et Fraser, 1993; Richard, Schneider et Mallet, 2012; Rutter, Maughan, Mortimore, Ouston et Smith, 1979) nous ont convaincus du besoin de doter les chercheurs et les praticiens d'instruments de mesure susceptibles de leur être utile pour mieux comprendre les facteurs en jeu dans la perception du climat scolaire et pour travailler à l'amélioration constante de ce climat. Nous avons donc traduit et adapté en langue française deux instruments de mesure du climat scolaire 
s'adressant aux élèves des niveaux élémentaires et intermédiaires, soit l'ICE et l'ICRC. L'étude de la validité de ces instruments auprès d'un échantillon d'élèves néobrunswickois donne des résultats satisfaisants en termes de construit théorique, c'est-à-dire, pour l'IEC, la mesure des quatre dimensions du climat scolaire que sont la collaboration, l'orientation vers les tâches, la participation et l'empathie de l'enseignant, et pour l'ICRC, la mesure des perceptions des élèves en ce qui a trait à la structuration et à la nature des activités qui leur sont proposées à l'extérieur de la classe. Les deux instruments ont également un niveau de fiabilité acceptable en termes de cohérence interne des items. Malgré le besoin de recherches plus approfondies dans ce domaine, les résultats de la présente validation transculturelle de l'ICE et de I'ICRC permettent d'en recommander une utilisation prudente aux fins de recherche et d'intervention dans les milieux scolaire où évoluent les élèves francophones en situation linguistique minoritaire.

Dans ce contexte, des instruments tels que l'ICE et l'ICRC pourraient contribuer à examiner les liens entre les dimensions du climat scolaire et les besoins sous-jacents à un vécu langagier autonomisant, notamment, les besoins d'autonomie, de compétence et d'appartenance (Landry, Allard, Deveau et Bourgeois, 2005; Landry, Deveau, Losier et Allard, 2009). De plus, selon Gilbert, LeTouzé, Thériault et Landry (2004), l'enseignant joue un rôle de premier ordre dans la conscientisation des jeunes francophones aux réalités d'un statut minoritaire, son empathie envers eux est donc d'une grande importance. Dans quelle mesure le climat scolaire contribue-t-il au vécu langagier autonomisant, à la formation de l'identité ethnolinguistique chez les élèves des niveaux élémentaires et intermédiaires, et, ultimement, à la motivation et au comportement langagier des jeunes francophones en situation minoritaire, voilà autant de questions à explorer.

\section{Références}

Aldridge, J.M., Fraser, B.J. et Sebela, M.P. (2004). Using teacher action research to promote constructivist learning environments in South Africa. South African Journal of Education, 24(4), 245-253.

Allard, R., Landry, R. et Deveau, K. (2005). Conscientisation ethnolangagière et comportement engagé en milieu minoritaire. Francophonies d'Amérique, 20 (automne), 95-109.

Allen, D. et Fraser, B.J. (2007). Parent and student perceptions of classroom learning environment and its association with student outcomes. Learning Environments Research, 10(1), 67-82.

Anastasi, A. et Urbina, S. (1997). Psychological testing (7th ed.). Upper Saddle River : Prentice-Hall.

Association canadienne d'éducation de langue française (2006). Cadre d'orientation en construction identitaire. Québec : ACELF.

Ciucci, E. et Smorti, A. (1998). II fenomeno delle pretonenze nella scuola: problemi e prospettive di intervento. [Le phénomène d'intimidation dans les écoles : Problèmes et pistes d'intervention]. Psichiatria dell'infanzia e dell'adolescenza, 65(2), 147157.

Cormier, M. (2005). La pédagogie en milieu minoritaire francophone : une recension des écrits. Moncton : Institut canadien de recherche sur les minorités linguistiques; Ottawa : Fédération canadienne des enseignants et des enseignantes.

Cormier, M., Pruneau, D. et Rivard, L. P. (2010). Améliorer les apprentissages en sciences en milieu francophone minoritaire : résultats de l'expérimentation d'un modèle pédagogique. Revue des sciences de l'éducation, 36(2), 343-363.

Cormier, C., Pruneau, D., Rivard, L. et Blain, S. (2004). Un modèle pédagogique pour améliorer l'apprentissage des sciences en milieu linguistique minoritaire. Francophonies d'Amérique, 18(1), 21-35.

Cossette, M. C., Potvin, P., Marcotte, D., Fortin, L., Royer, É. et Leclerc, D. (2004). Le risque de décrochage scolaire et la perception du climat de classe chez les élèves du secondaire. Revue de psychoéducation, 33(1), 117-136.

Dempster, F. N. (1988). The spacing effect: A case study in the failure to apply the results of psychological research. American Psychologist, 43(8), 627-634.

Diaz, M. E. (1983). Adaptation et validation d'une échelle pour mesurer le climat social des cours au niveau de l'enseignement secondaire. Bulletin de psychologie scolaire et d'orientation, 32(3), 99-111. 
Espelage, D.L. et Swearer, S. M. (Dir.). (2004). Bullying in american schools : A social-ecological perspective on prevention and intervention. Mahwah : Lawrence Erlbaum.

Espelage, D. L. et Swearer, S. M. (2010). A social-ecological model for bullying prevention and intervention: Understanding the impact of adults in the social ecology of youngsters. Dans S. R. Jimerson, S. Swearer et D. Espelage (Dir.), Handbook of bullying in schools : An international perspective (p. 61-72). New York : Routledge/Taylor \& Francis Group.

Fisher, D., Grady, N. et Fraser, B.J. (1995). Associations between school-level and classroom-level environment. International Studies in Educational Administration, 25(3), 1-15.

Fraser, B. J. (1989).Twenty years of classroom climate work : Progress and prospect. Journal of Curriculum Studies, 21(4), 307-327.

Fraser, B. J. (1994). Research on classroom and school climate. Dans D. Gabel (Dir.), Handbook of research on science teaching and learning (p. 493-541). New York : Macmillan.

Fraser, B. J. (1998). Classroom environment instruments : Development, validity and application. Learning Environments Research, 1(1), 7-33.

Fraser, B. J. et Fisher, D. L. (1983). Student achievement as a function of person-environment fit: A regression surface analysis. British Journal of Educational Psychology, 53(1), 89-99.

Gilbert, A., Le Touzé, S., Thériault, J. Y. et Landry, R. (2004). Le personnel enseignant face aux défis de l'enseignement en milieu minoritaire francophone. Ottawa : Fédération canadienne des enseignantes et des enseignants, Centre interdisciplinaire de recherche sur la citoyenneté et les minorités; Moncton : Institut canadien de recherche sur les minorités linguistiques.

Haertel, G. D., Walberg, H. J. et Haertel, E. H. (1981). Socio-psychological environments and learning : A quantitative synthesis. British Educational Research Journal, 7(1), 27-36.

Halpin, A. W. et Croft, D. B. (1963). Organizational climate of schools. Chicago : Midwest Administration Center.

Hambleton, R. K. (1994). Guidelines for adapting educational and psychological tests : A progress report. European Journal of Psychological Assessment, 10(3), 229-244.

Hunt, D. E. (1975). Person-environment interaction: A challenge found wanting before it was tried. Review of Educational Research, 45(2), 209-230.

Janosz, M., Georges, P. et Parent, S. (1998). L'environnement socioéducatif à l'école secondaire : un modèle théorique pour guider l'évaluation du milieu. Revue canadienne de psycho-éducation, 27(2), 285-306.

Landry, R., Allard, R., Deveau, K. et Bourgeois, N. (2005). Autodétermination du comportement langagier en milieu minoritaire : un modèle conceptuel. Francophonies d'Amérique, 20 (automne), 63-78.

Landry, R., Deveau, K., Losier, G. et Allard, R. (2009). Identité ethnolinguistique, autodétermination et satisfaction de vie en contexte francophone minoritaire. Francophonies d'Amérique, 28 (automne), 47-70.

Laveault, D. et Grégoire, J. (2002). Introduction aux théories des tests : en psychologie et en sciences de l'éducation ( $2^{\mathrm{e}}$ éd.). Bruxelles : De Boeck Université.

LeBlanc, J. et Beaton, A.M. (2011). Les facteurs qui influent sur le rendement à une tâche en français chez les femmes d'origine acadienne : évocation de catégories sociales et confiance dans son rendement. Éducation francophone en milieu minoritaire, 6(1), 1-15.

Leff, S. S., Power, T. J. et Goldstein, A. B. (2004). Outcome measures to assess the effectiveness of bullying-prevention programs in the schools. Dans D. L. Espelage et S. M. Swearer (Dir.), Bullying in American schools : A social-ecological perspective on prevention and intervention (p. 269-293). Mahwah : Lawrence Erlbaum.

Leff, S. S., Power, T. J., Costigan, T. E. et Manz, P. H. (2003). Assessing the climate of the playground and lunchroom : Implications for bullying prevention programming. School Psychology Review, 32(3), 418-430.

McRobbie, C. J. et Fraser, B. J. (1993). Associations between student outcomes and psychosocial science environment. Journal of Educational Research, 87(2), 78-85.

Melton, G. B., Limber, S. P., Cunningham, P., Osgood, D. W., Chambers, J., Flerx, V., Henggeler, S. et Nation, M. (1998). Violence among rural youth (Final report). Washington : Office of Juvenile Justice and Delinquency Prevention.

Mortimore, P. (1995). The positive effects of schooling. Dans M. Rutter (Dir.), Psychosocial disturbances in young people: Challenges for prevention (p. 333-363). New York : Cambridge University Press.

Moos, R. H. (2002). The mystery of human context and coping : An unraveling of clues. American Journal of Community Psychology, 30(1), 67-88.

Pellegrini, A. D. (1995). School recess and playground behavior : Educational and developmental roles. Albany : State University of New York Press.

Pellegrini, A. D. et Bjorklund, D. F. (1996). The place of recess in school : Issues in the role of recess in children's education and development. Journal of Research in Childhood Education, 11(1), 5-13. 
Pellegrini, A. D. et Davis, P. D. (1993). Relations between children's playground and classroom behaviour. British Journal of Educational Psychology, 63(1), 88-95.

Peterson, R. L. et Skiba, R. (2000). Creating school climates that prevent school violence. Preventing School Failure, 44(3), 122-129.

Richard, J.F., Schneider, B.H. et Mallet, P. (2012). Revisiting the whole-school approach to bullying : Really looking at the whole school. School Psychology International, 33(3), 263-284.

Rutter, M., Maughan, B., Mortimore, P., Ouston, J. et Smith, A. (1979). Fifteen Thousand Hours: Secondary Schools and their effects on Children. London : Open Books.

Sinclair, B. B. et Fraser, B. J. (2002). Changing classroom environments in urban middle schools. Learning Environments Research, 5(3), 301-328.

Stevens, V., De Bourdeaudhuij, I. et Van Oost, P. (2000). Bullying in Flemish schools : An evaluation of anti-bullying intervention in primary and secondary schools. British Journal of Educational Psychology, 70(2), 195-210.

Vallerand, R. J. (1989). Vers une méthodologie de validation trans-culturelle de questionnaires psychologiques : Implications pour la recherche en langue française. Psychologie canadienne, 30(4), 662-680.

Zullig, K., Huebner, E. S. et Patton, J. M. (2011). Relationships among school climate domains and school satisfaction. Psychology in the Schools, 48(2), 133-145. 